\title{
Diagnostic value of fibrinogen to prealbumin ratio and gamma-glutamyl transpeptidase to platelet ratio in the progression of AFP-negative hepatocellular carcinoma
}

\author{
Li Huang ${ }^{1}$, Zhuning Mo², Zuojian Hu${ }^{1}$, Linyan Zhang ${ }^{1}$, Shanzi Qin ${ }^{1}$, Xue Qin ${ }^{1}$ and Shan $\mathrm{Li}^{\text {* }^{*}}$
}

\begin{abstract}
Background: This study aimed to comprehensively assess the diagnostic value of fibrinogen to prealbumin ratio (FPR) and gamma-glutamyl transpeptidase to platelet ratio (GPR) as single markers or in combination in patients with alpha-fetoprotein-negative (AFP-negative) hepatocellular carcinoma (HCC).

Methods: A total of 199 healthy controls and 515 AFP-negative patients were enrolled in this study, including 180 HCC inpatients, 151 liver cirrhosis (LC) patients, and 184 chronic hepatitis (CH) cases. Mann-Whitney U or Kruskal-Wallis $\mathrm{H}$ test were used to analyze differences between groups in laboratory parameters and clinicopathological features. The diagnostic value of FPR and GPR, alone or in combination, in AFP-negative HCC (AFP-NHCC) patients was determined via a receiver operating characteristic $(R O C)$ curve.

Results: The levels of FPR and GPR were gradually increased in the development of AFP-NHCC and positively correlated with the tumor size and Barcelona Clinic Liver Cancer (BCLC) stages. Moreover, GPR was associated with Edmondson-Steiner grades. After univariate logistic regression analysis, FPR and GPR remained independent predictors of adverse outcomes. The combination of FPR and GPR had a good ability to detect AFP-NHCC from the control group (area under curve $[A \cup C]=0.977)$, AFP-negative $C H(A \cup C=0.745)$, and AFP-negative LC (AUC=0.666). FPR combined with GPR possessed a larger area $(0.943,0.971)$ and sensitivity $(87.50 \%, 89.81 \%)$ than FPR or GPR alone for differentiating AFP-NHCC with tumor size $<3 \mathrm{~cm}$ or at the BCLC-A stage.
\end{abstract}

Conclusions: The pretreatment levels of FPR and GPR played vital roles in the development of AFP-NHCC, especially in patients with early or small AFP-NHCC.

Keywords: Fibrinogen to prealbumin ratio, Gamma-glutamyl transpeptidase to platelet ratio, Diagnostic, AFPnegative hepatocellular carcinoma

\footnotetext{
*Correspondence: lis8858@126.com

${ }^{1}$ Department of Clinical Laboratory, First Affiliated Hospital of Guangxi

Medical University, Nanning 530021, Guangxi Zhuang Autonomous Region, China

Full list of author information is available at the end of the article
}

\section{Background}

Hepatocellular carcinoma $(\mathrm{HCC})$ is the sixth-most commonly diagnosed cancer and the fourth leading cause of tumor-related death worldwide in 2018 [1]. Accumulated evidence demonstrates that inefficient diagnosis of HCC is still a major cause of high mortality, especially in patients harboring early or small HCC [2]. Surveillance guidelines for patients with a high

(c) The Author(s) 2020. This article is licensed under a Creative Commons Attribution 4.0 International License, which permits use, sharing, adaptation, distribution and reproduction in any medium or format, as long as you give appropriate credit to the original author(s) and the source, provide a link to the Creative Commons licence, and indicate if changes were made. The images or other third party material in this article are included in the article's Creative Commons licence, unless indicated otherwise in a credit line to the material. If material is not included in the article's Creative Commons licence and your intended use is not permitted by statutory regulation or exceeds the permitted use, you will need to obtain permission directly from the copyright holder. To view a copy of this licence, visit http://creativeco mmons.org/licenses/by/4.0/. The Creative Commons Public Domain Dedication waiver (http://creativecommons.org/publicdomain/ zero/1.0/) applies to the data made available in this article, unless otherwise stated in a credit line to the data. 
risk of developing $\mathrm{HCC}$ primarily relied on ultrasound imaging and alpha-fetoprotein (AFP) [3]. Currently, serum AFP remains the most important and commonly serological diagnostic biomarker, but about $30-40 \%$ of overall HCC patients have normal AFP levels $(<20 \mathrm{ng} /$ $\mathrm{mL}$ ) [4]. This is referred to as AFP-negative hepatocellular carcinoma (AFP-NHCC) [5]. Even though the proportion of AFP-negative was as high as $15-30 \%$ in advanced patients, the American Association for the Study of Liver Diseases updated their practice guidelines in 2011, saying that AFP was no longer recommended for the detection of early HCC [6]. Although imaging technology has greatly improved the level of HCC detection, ultrasound images often fail to recognize small HCC nodules or distinguish malignant nodules from benign ones $[7,8]$, and the diagnosis rate for patients with AFP-NHCC is only 10.4\% [9]. Patients with AFP-NHCC often have mild clinical symptoms, so other tumor markers are indispensable for its diagnosis, especially in the early stage of the disease. An array of numerous alternative yet costly tumor markers have been proposed to screen liver cancer, including Golgi protein 73 (GP73) [10], glypican-3 (GPC-3) [11], protein induced by vitamin $\mathrm{K}$ absence or antagonist-II (PIVKAII) [12], and some MicroRNAs [13]. However, none have shown sufficient sensitivity and/or specificity to meet the clinical routine practice requirements for the early diagnosis HCC [14]. Therefore, novel biomarkers with more economical, accurate, and useful predictions for the early diagnosis of $\mathrm{HCC}$ are urgently needed, especially for AFP-negative patients.

An increasing amount of research has pinpointed systematic inflammation and abnormal metabolism involved in the diagnosis and progression of HCC, such as fibrinogen (Fib) [15], prealbumin (PA) [16], platelet (PLT) [17], gamma-glutamyl transferase (GGT) [18], and combinations of several single markers in the forms of ratios [19, 20]. Indeed, Fib promotes the synthesis of proinflammatory cytokines and fibroblast growth factors to induce the malignant proliferation of tumor cells and accumulates vascular endothelium to enhance tumor angiogenesis [21, 22]. Zhang et al. [23] reported that hyperfibrinogenemia is relevant to advanced tumor stages and poor survival in patients with HCC. PA has a short half-life as a predictor of inflammatory stress and nutritional status [24], making it an effective biomarker for morbidity, mortality and tumor progression [25]. Therefore, fibrinogen to prealbumin ratio (FPR), which combines Fib and PA, has been used to predict clinical efficacy and outcome for several types of human cancers $[20,26]$. In addition, the preoperative FPR can independently predict recurrencefree survival and overall survival and help identify HCC patients who could benefit from adjuvant chemotherapy
[27]. Therefore, FPR may be a potent prognostic indicator for AFP-NHCC patients.

Gamma-glutamyl transpeptidase (GGT) to platelet ratio (GPR) was proposed by Lemoine et al. as a novel predictor for liver disease [28]. Ample evidence suggested that GPR was a good predictor for the diagnosis and prognosis of hepatitis liver, hepatic fibrosis, and hepatocellular carcinoma. Wang et al. [29] pointed out that the sensitivity and specificity of GPR in diagnosing liver inflammation was as high as $83.47 \%$ and $61.33 \%$, respectively. The GPR showed better diagnostic accuracy than the aspartate transaminase to platelet ratio index (APRI) and the fibrosis index based on four factors (FIB4 ) in assessing liver fibrosis in chronic hepatitis B (CHB) patients in West African populations [28]. Ke et al. [30] reported that GPR could predict complications in HCC patients undergoing minor liver resection. Hence, we hypothesized that this biomarker might have a better diagnostic value in the progression of AFP-NHCC.

To date, several studies have researched the value of FPR and GPR in the prognosis of HCC. However, there is a lack of data about the diagnostic value of FPR and GPR for the development of AFP-NHCC patients. Thus, this study evaluates whether the FPR and GPR could be used as predictive markers for patients with AFP-NHCC.

\section{Material and method \\ Patients}

$180 \mathrm{HCC}$ patients, $184 \mathrm{CH}$ patients, and $151 \mathrm{LC}$ patients who were all AFP-negative $(<20 \mathrm{ng} / \mathrm{mL})$ were continuously recruited from the First Affiliated Hospital of Guangxi Medical University between Jan 2012 and Oct 2019. The inclusion criteria of AFP-negative patients were as follows: (1) newly diagnosed with HCC, and verified by surgical histopathology examination; LC was diagnosed via pathological examination and typical morphology upon ultrasonography or computed tomography (CT) imaging; or $\mathrm{CH}$ patients were confirmed to have been infected with hepatitis B virus (HBV)/hepatitis C virus $(\mathrm{HCV})$ for at least 6 months; (2) no blood-system diseases or immunity-related diseases; (3) no presence of other types of cancers; (4) no infectious diseases other than hepatitis B or C; and (5) no organic disease outside of the liver. We also enrolled 199 healthy individuals with no history of cancer and no clinical evidence of liver disease as a control group. This research was approved by the Ethics Committee of the First Affiliated Hospital of Guangxi Medical University, and informed consent was obtained from all the participants.

A total of 180 patients (range 28-78 years) with AFPNHCC were recruited in this study, including 151 patients with positive hepatitis B surface antigen, 21 patients with hepatitis $C$ virus positive liver diseases, and 
8 subjects with other types of liver cancer. And serum HBV-DNA levels $\geq 500 \mathrm{IU} / \mathrm{mL}$ was achieved in 69 of 151 AFP-NHCC patients who were hepatitis B surface antigen positive. According to the Barcelona Clinic Liver Cancer (BCLC) staging system, 108 patients (60.0\%) had stage A, 53 (29.4\%) had stage B, and 19 (10.6\%) had stage $C$. Of the 151 cirrhosis patients, 130 were positive for hepatitis B surface antigen, 10 were positive for hepatitis $C$ virus antibody, and 11 were other causes of cirrhosis, respectively. Subjects with hepatitis B surface antigen-positive were 159 in AFP-negative $\mathrm{CH}$ group, the rest were hepatitis $C$ virus antibody-positive patients $(25 / 184)$.

\section{Data detection and acquisition}

All of the corresponding data for this study was extracted from the hospital's electronic medical records before each qualified participant's operation, including sex, age, white blood cells (WBC), platelets (PLT), hemoglobin $(\mathrm{Hb})$, fibrinogen (Fib), prealbumin (PA), alpha-fetoprotein (AFP), alkaline phosphatase (ALP), total bilirubin (TBIL), alanine aminotransferase (ALT), aspartate amino transferase (AST), and gamma-glutamyl transpeptidase (GGT). Plasma fibrinogen concentrations were determined by the Clauss method using a Sysmex CA7000 automatic coagulation analyzer. Blood tests were performed with a Beckman-Coulter LH 780 hematology analyzer (Beckman Coulter, Brea, CA). The levels of PA, AST, ALT, TBIL, ALP, and GGT were tested by a Hitachi 7600 automatic biochemical analyzer (Tokyo, Japan) produced by Shanghai Zhicheng Bio-Technology Co., Ltd. Hepatitis B surface antigens and hepatitis $C$ antibodies were analyzed using a chemiluminescent immunoassay (Abbott GmBH Diagnostika, Wiesbaden-Delkenheim, Germany). The values of FPR and GPR were calculated using the following formulas: $\mathrm{FPR}=$ fibrinogen level/prealbumin level; GPR = gamma-glutamyl transpeptidase level/platelet count.

\section{Statistical analysis}

None of the data met the criteria for a normal distribution based on a Kolmogorov-Smirnov test. The median and interquartile ranges were applied for non-normally distributed data. The Mann-Whitney U test or KruskalWallis $\mathrm{H}$ test were conducted to detect differences between groups in laboratory parameters and clinical characteristics. A receiver operating characteristic (ROC) curve and the area under the curve (AUC) were determined by MedCalc statistical software (version 18.1.1). The SPSS16.0 statistical software package was used for data processing and analysis, using a significant level of 0.05 .

\section{Result}

\section{General information about study population}

Basic information is shown in Table 1. The median PA of the 151 patients with AFP-negative LC was lower than those in other groups. Similarly, corresponding differences appeared in the levels of circulating PLT, WBC, $\mathrm{Hb}$, and Fib. On the contrary, AFP- negative LC patients had higher TBIL and AST compared with other individuals. The value of FPR and GPR gradually increased with the development of AFP-NHCC $(p<0.001)$ (Fig. 1).

\section{Correlation between FPR, GPR, and clinicopathological features in AFP-NHCC}

As shown in Table 2, FPR and GPR were both associated with Barcelona Clinic Liver Cancer (BCLC) stages, but not related to the Child-Pugh grade, tumor encapsulation, or tumor multiplicity (all $p>0.05$ ). Although 22 specimens were not clearly classified by Edmondson-Steiner grade, GPR showed a significant difference between the three subgroups of AFP-NHCC stages $(p=0.032)$.

\section{Logistic regression used to distinguish AFP-NHCC from controls}

As shown in Table 3, the correlation between AFPNHCC and some potential risk factors were analyzed using binary logistic regression, such as gender, age, TBIL, AST, ALT, ALP, FPR, and GPR. In the univariate analysis,"whether suffering from AFP-NHCC" was the response variable, these potential risk parameters were used as corresponding input variables one by one. In consideration of other confounding factors and the impact of a suppressor effect, variables with a significant value of $p<0.05$ were subjected to a multivariate analysis and further screened by enter method to evaluate their independent effect. Odd ratio (OR) and 95\% confidence interval (CI) were also calculated for each other. After the univariate analysis, several significant parameters were chosen as potential independent predictors for further multivariate analysis, including TBIL $(\mathrm{OR}=1.086$, 95\% $\mathrm{CI}=1.039-1.134, \quad p<0.001), \quad$ ALT $\quad(\mathrm{OR}=1.133$, $95 \% \mathrm{CI}=1.101-1.167, \quad p<0.001), \quad$ AST $\quad(\mathrm{OR}=1.232$, $95 \% \mathrm{CI}=1.175-1.293, \quad p<0.001), \quad$ ALP $\quad(\mathrm{OR}=1.023$, 95\% $\mathrm{CI}=1.013-1.033, p<0.001)$, FPR $(\mathrm{OR}=2.082,95 \%$ $\mathrm{CI}=1.783-2.430, p<0.001)$, and GPR $\left(\mathrm{OR}=1.748 \times 10^{8}\right.$, $\left.95 \% \mathrm{CI}=2.003 \times 10^{6}-1.525 \times 10^{10}, \quad p<0.001\right) . \quad$ After adjusting for these six predictors, the results of the analysis demonstrated that FPR $(\beta=0.841, p<0.001)$, GPR $(\beta=15.927, p<0.001)$, and AST $(\beta=0.078, p=0.023)$ were still important indicators closely related to the occurrence of AFP-NHCC. The optimal model for distinguishing AFP-NHCC patients from the control group 


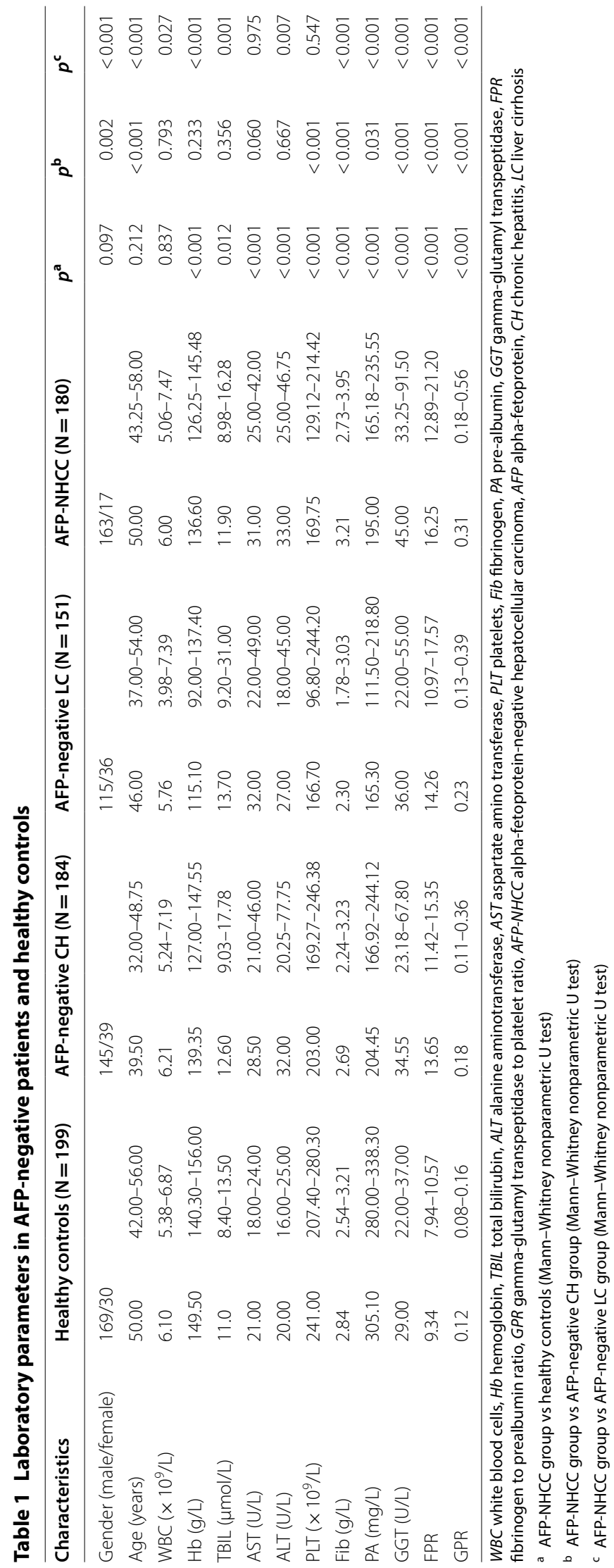



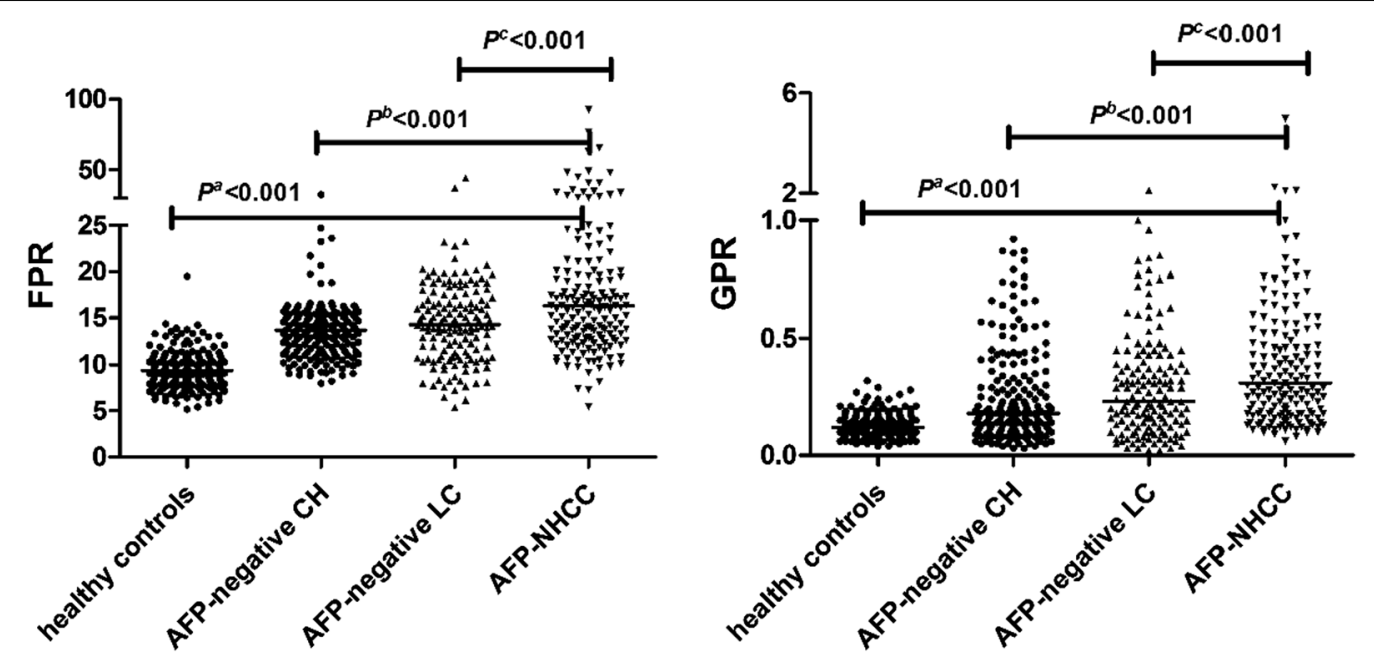

Fig. 1 FPR and GPR among four groups. FPR fibrinogen to prealbumin ratio, GPR gamma-glutamyl transpeptidase to platelet ratio, AFP-NHCC alpha-fetoprotein-negative hepatocellular carcinoma, AFP alpha-fetoprotein, CH chronic hepatitis, LC liver cirrhosis. $p^{\text {a. }}$ AFP-NHCC group vs healthy controls; $p^{\text {b. }}$ : AFP-NHCC group vs AFP-negative CH group; $p^{c}$ : AFP-NHCC group vs AFP-negative LC group

Table 2 Correlation between clinicopathological features and FPR and GPR in AFP-NHCC

\begin{tabular}{|c|c|c|c|c|c|}
\hline & Number (\%) & FPR & $p$ & GPR & $p$ \\
\hline \multicolumn{6}{|c|}{ Edmondson-Steiner grade } \\
\hline । & $19(10.6)$ & $13.56(11.27-26.66)$ & \multirow[t]{4}{*}{0.345} & $0.47(0.33-0.79)$ & \multirow[t]{4}{*}{0.032} \\
\hline$\|$ & $114(63.3)$ & $15.99(13.06-21.11)$ & & $0.300(0.17-0.59)$ & \\
\hline III & $25(13.9)$ & $17.27(14.07-21.84)$ & & $0.300(0.19-0.50)$ & \\
\hline Unknown & $22(12.2)$ & 16.94 (13.59-20.38) & & $0.27(0.14-0.39)$ & \\
\hline \multicolumn{6}{|l|}{ BCLC stage } \\
\hline$A$ & $108(60.0)$ & 15.65 (12.54-19.03) & \multirow[t]{2}{*}{0.004} & $0.29(0.17-0.48)$ & \multirow[t]{2}{*}{0.024} \\
\hline$B-C$ & $72(40.0)$ & $17.14(13.86-27.02)$ & & $0.37(0.18-0.73)$ & \\
\hline \multicolumn{6}{|c|}{ Child-Pugh grade } \\
\hline$A$ & $162(90.0)$ & 16.12 (12.79-20.35) & \multirow[t]{2}{*}{0.334} & $0.30(0.18-0.54)$ & \multirow[t]{2}{*}{0.137} \\
\hline$B-C$ & $18(10.0)$ & $17.00(12.93-33.08)$ & & $0.42(0.24-0.69)$ & \\
\hline \multicolumn{6}{|c|}{ Tumor size $(\mathrm{cm})$} \\
\hline$<3$ & $40(22.2)$ & $14.22(11.52-16.84)$ & \multirow[t]{2}{*}{0.001} & $0.22(0.13-0.30)$ & \multirow[t]{2}{*}{$<0.001$} \\
\hline$\geq 3$ & $140(77.8)$ & $16.99(13.63-23.75)$ & & $0.37(0.19-0.62)$ & \\
\hline \multicolumn{6}{|c|}{ Tumor encapsulation } \\
\hline Complete & $123(68.3)$ & 16.30 (12.74-21.24) & \multirow[t]{2}{*}{0.721} & $0.31(0.18-0.55)$ & \multirow[t]{2}{*}{0.384} \\
\hline None & $57(31.7)$ & 15.37 (13.22-21.59) & & $0.34(0.19-0.62)$ & \\
\hline \multicolumn{6}{|c|}{ Tumor multiplicity } \\
\hline Single & 167 (92.8) & 16.30 (12.80-21.27) & \multirow[t]{2}{*}{0.471} & $0.32(0.18-0.57)$ & \multirow[t]{2}{*}{0.437} \\
\hline Multiple & $13(7.2)$ & $14.07(13.81-18.34)$ & & $0.27(0.17-0.41)$ & \\
\hline
\end{tabular}

FPR fibrinogen to prealbumin ratio, GPR gamma-glutamyl transpeptidase to platelet ratio, AFP-NHCC alpha-fetoprotein-negative hepatocellular carcinoma, $B C L C$ Barcelona Clinic Liver Cancer

was established through integration (logit $P=0.841 \times$ $\mathrm{FPR}+15.927 \times \mathrm{GPR}+0.078 \times \mathrm{ALT}-17.909)$. For this model, the AUC value was 0.981 (0.961 to 0.992), while the sensitivity and specificity were $92.22 \%$ and $97.99 \%$, respectively.
Evaluating the diagnostic value of FPR, GPR between AFP-NHCC and other subjects

The results of the ROC curve analysis are shown in Table 4 and Fig. 2. The AUC value of FPR $(\mathrm{AUC}=0.935)$ and GPR $(\mathrm{AUC}=0.884)$ had a good diagnostic ability for 
Table 3 Univariate and multivariate analyses used for differentiating significant predictors to distinguish AFP-NHCC from healthy controls

\begin{tabular}{|c|c|c|c|c|c|c|}
\hline \multirow[t]{2}{*}{ Variables } & \multicolumn{3}{|c|}{ Univariate analysis } & \multicolumn{3}{|c|}{ Multivariate analysis } \\
\hline & OR & $95 \% \mathrm{Cl}$ & $P$-value & OR & $95 \% \mathrm{Cl}$ & $p$-value \\
\hline Gender & 0.588 & $0.312-1.106$ & 0.099 & & & \\
\hline Age(y) & 1.013 & $0.992-1.035$ & 0.232 & & & \\
\hline TBIL & 1.086 & $1.039-1.134$ & $<0.001$ & 1.110 & $0.985-1.250$ & 0.086 \\
\hline AST & 1.232 & $1.175-1.293$ & $<0.001$ & 1.079 & $0.969-1.200$ & 0.166 \\
\hline ALT & 1.133 & $1.101-1.167$ & $<0.001$ & 1.081 & $1.011-1.157$ & 0.023 \\
\hline ALP & 1.023 & $1.013-1.033$ & $<0.001$ & 0.997 & $0.970-1.024$ & 0.806 \\
\hline FPR & 2.082 & $1.783-2.430$ & $<0.001$ & 2.320 & $1.824-2.949$ & $<0.001$ \\
\hline GPR & $1.748 \times 10^{8}$ & $2.003 \times 10^{6}-1.525 \times 10^{10}$ & $<0.001$ & $8.260 \times 10^{6}$ & $5.031 \times 10^{3}-1.356 \times 10^{10}$ & $<0.001$ \\
\hline
\end{tabular}

$T B I L$ total bilirubin, $A L T$ alanine aminotransferase, $A S T$ aspartate amino transferase, $A L P$ alkaline phosphatase, FPR fibrinogen to pre-albumin ratio, GPR gammaglutamyl transpeptidase to platelet ratio, CI confidence interval, OR odd ratio, AFP-NHCC alpha-fetoprotein-negative hepatocellular carcinoma

Table 4 Diagnostic efficacy of FPR and GPR used alone or in combination in differentiating patients with AFP-NHCC from other patients

\begin{tabular}{|c|c|c|c|c|c|c|c|c|c|}
\hline & Cutoff & Sensitivity (\%) & Specificity (\%) & PLR & NLR & PPV (\%) & NPV (\%) & AUC $(95 \% \mathrm{CI})$ & $p$ \\
\hline $\mathrm{FPR}^{\mathrm{a}}$ & 11.57 & 86.67 & 88.44 & 7.50 & 0.15 & 87.2 & 88.0 & $0.935(0.905-0.957)$ & $<0.001$ \\
\hline $\mathrm{GPR}^{\mathrm{a}}$ & 0.21 & 67.78 & 94.47 & 12.26 & 0.34 & 91.7 & 76.4 & $0.884(0.848-0.915)$ & $<0.001$ \\
\hline $\mathrm{FPR}^{\mathrm{a}}+\mathrm{GPR}^{\mathrm{a}}$ & 0.44 & 91.11 & 96.48 & 25.90 & 0.09 & 95.9 & 92.3 & $0.977(0.957-0.990)$ & $<0.001$ \\
\hline $\mathrm{FPR}^{\mathrm{b}}$ & 16.70 & 46.11 & 94.02 & 7.71 & 0.57 & 88.3 & 64.1 & $0.696(0.646-0.743)$ & $<0.001$ \\
\hline$G P R^{b}$ & 0.23 & 64.44 & 61.96 & 1.69 & 0.57 & 62.4 & 64.0 & $0.677(0.626-0.725)$ & $<0.001$ \\
\hline$F P R^{b}+G P R^{b}$ & 0.55 & 67.22 & 74.46 & 2.63 & 0.44 & 72.0 & 69.9 & $0.745(0.697-0.789)$ & $<0.001$ \\
\hline $\mathrm{FPR}^{\mathrm{C}}$ & 20.72 & 26.11 & 93.38 & 3.94 & 0.79 & 82.5 & 51.5 & $0.623(0.568-0.675)$ & $<0.001$ \\
\hline $\mathrm{GPR}^{\mathrm{C}}$ & 0.20 & 68.89 & 43.71 & 1.22 & 0.71 & 59.3 & 54.1 & $0.617(0.563-0.670)$ & $<0.001$ \\
\hline$F P R^{c}+G P R^{c}$ & 0.42 & 46.11 & 81.46 & 2.49 & 0.66 & 74.8 & 55.9 & $0.666(0.612-0.716)$ & $<0.001$ \\
\hline $\mathrm{FPR}^{\mathrm{d}}$ & 11.30 & 80.00 & 86.43 & 5.90 & 0.23 & 54.2 & 95.6 & $0.914(0.871-0.946)$ & $<0.001$ \\
\hline $\mathrm{GPR}^{\mathrm{d}}$ & 0.19 & 60.00 & 89.95 & 5.97 & 0.44 & 54.5 & 91.8 & $0.801(0.745-0.850)$ & $<0.001$ \\
\hline$F P R^{d}+G P R^{d}$ & 0.15 & 87.50 & 86.93 & 6.70 & 0.14 & 57.4 & 97.2 & $0.943(0.906-0.969)$ & $<0.001$ \\
\hline $\mathrm{FPR}^{\mathrm{e}}$ & 11.26 & 86.11 & 85.93 & 6.12 & 0.16 & 76.9 & 91.9 & $0.916(0.879-0.944)$ & $<0.001$ \\
\hline $\mathrm{GPR}^{\mathrm{e}}$ & 0.21 & 66.67 & 94.47 & 12.06 & 0.35 & 86.7 & 83.9 & $0.859(0.814-0.896)$ & $<0.001$ \\
\hline $\mathrm{FPR}^{\mathrm{e}}+\mathrm{GPR}^{\mathrm{e}}$ & 0.16 & 89.81 & 96.48 & 25.53 & 0.11 & 93.3 & 94.6 & $0.971(0.945-0.987)$ & $<0.001$ \\
\hline
\end{tabular}

FPR fibrinogen to prealbumin ratio, GPR gamma-glutamyl transpeptidase to platelet ratio, $P L R$ positive likelihood ratio, NLR negative likelihood ratio, $P P V$ positive predictive value, NPV negative predictive value, Cl confidence interval, AUC area under curve, AFP-NHCC alpha-fetoprotein-negative hepatocellular carcinoma, AFP alpha-fetoprotein, $C H$ chronic hepatitis, $L C$ liver cirrhosis, BCLC Barcelona Clinic Liver Cancer

a AFP-NHCC patients vs healthy controls

b AFP-NHCC patients vs AFP-negative $\mathrm{CH}$ patients

c AFP-NHCC patients vs AFP-negative LC patients

d AFP-NHCC patients with tumor size $<3 \mathrm{~cm}$ vs healthy controls

e AFP-NHCC patients with BCLC-A stage vs healthy controls

distinguishing AFP-NHCC patients from controls. The sensitivity and specificity of the combination of FPR and GPR was increased to $91.11 \%$ and $96.48 \%$, respectively. Compared to healthy controls, the AUC value of combination for FPR and GPR was 0.977 (95\% CI $=0.957-$ 0.990 , positive likelihood ratio $[\mathrm{PLR}]=25.90$, negative likelihood ratio $[N L R]=0.09$ ), positive predictive value $[\mathrm{PPV}]=95.9 \%$, negative predictive value $[\mathrm{NPV}]=92.3 \%$ ).
Meanwhile, the AUC value of the combination of FPR and GPR in AFP-NHCC patients with tumor size $<3 \mathrm{~cm}$ was $0.943(95 \% \mathrm{CI}=0.906-0.969)$, while the sensitivity and specificity was $87.50 \%$ and $86.93 \%$, respectively. In the BCLC-A stage, the values of AUC, sensitivity, and specificity were $0.971,89.81 \%$, and $96.48 \%$, respectively.

To predict AFP-NHCC, the optimal cut-offs of FPR were 16.70 and 20.72, for the diagnosis of 


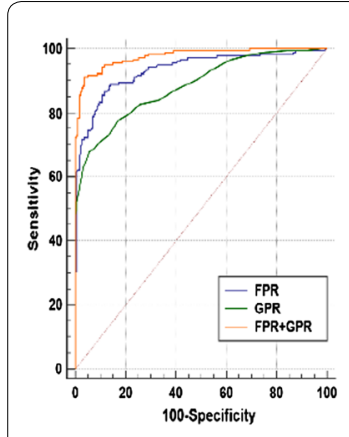

a

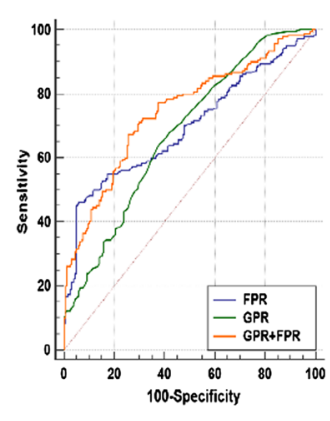

b

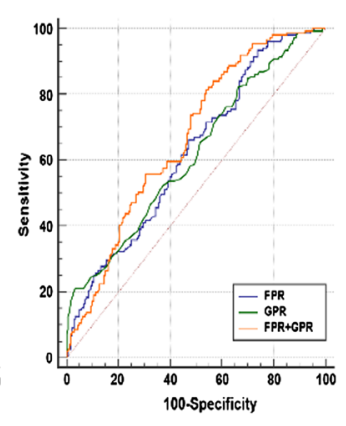

C

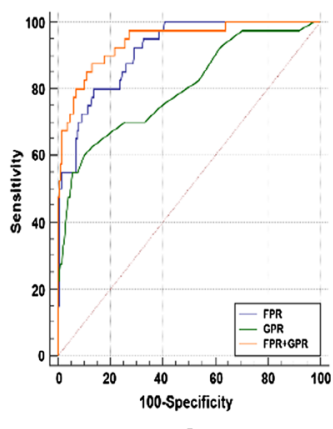

d

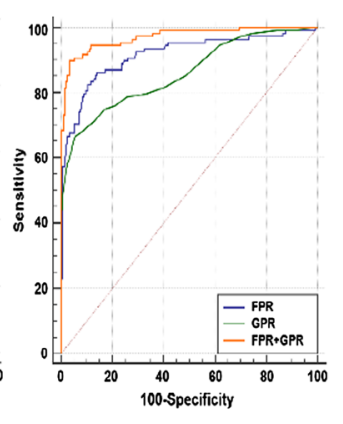

e

Fig. 2 The diagnostic value of FPR and GPR for distinction between AFP-NHCC patients from other groups. a AFP-NHCC patients vs healthy controls. b AFP-NHCC patients vs AFP-negative CH patients. c AFP-NHCC patients vs AFP-negative LC patients. d AFP-NHCC patients with tumor size $\leq 3 \mathrm{~cm}$ vs healthy controls. e AFP-NHCC patients with BCLC-A stage vs healthy controls. FPR fibrinogen to prealbumin ratio, GPR gamma-glutamyl transpeptidase to platelet ratio, AFP-NHCC alpha-fetoprotein-negative hepatocellular carcinoma, AFP alpha-fetoprotein, $C H$ chronic hepatitis, LC liver cirrhosis, BCLC Barcelona Clinic Liver Cancer

AFP-negative $\mathrm{CH} \quad(\mathrm{AUC}=0.696$, sensitivity $=46.11 \%$, specificity $=94.02 \%, \quad \mathrm{PPV}=88.3 \%$, and $\mathrm{NPV}=64.1 \%)$, and AFP-negative $\mathrm{LC}(\mathrm{AUC}=0.623$, sensitivity $=26.11 \%$, specificity $=93.38 \%, \quad \mathrm{PPV}=82.5 \%$, and $\mathrm{NPV}=51.5 \%)$, respectively. The optimal cut-offs of GPR were 0.23 and 0.20 , for the diagnosis of AFP-negative $\mathrm{CH}$ ( $\mathrm{AUC}=0.677$, sensitivity $=64.44 \%$, specificity $=61.96 \%, \quad P P V=62.4 \%$, and $\mathrm{NPV}=64.0 \%)$, and AFP-negative $\mathrm{LC}(\mathrm{AUC}=0.617$, sensitivity $=68.89 \%, \quad$ specificity $=43.71 \%, \quad P P V=59.3 \%$, and $\mathrm{NPV}=54.1 \%$ ), respectively. For the diagnosis of AFP-NHCC, the combined use of FPR and GPR resulted in a greater AUC (0.745 for AFP-negative $\mathrm{CH}$ and 0.666 for AFP-negative LC) than FPR or GPR alone.

\section{Discussion}

Early diagnosis of $\mathrm{HCC}$ is closely associated with its prognosis, which can substantially enhance the 5-year survival rate of patients [31]. AFP, as a regulatory surveillance indicator of $\mathrm{HCC}$, is limited in detecting $\mathrm{HCC}$ by poor diagnostic efficiency [32]. Thus, novel tumor biomarkers should be made to help the clinical diagnosis of AFP-NHCC. Numerous inflammatory response markers have been presented as effective, economical, and reliable indicators for the diagnosis and prognosis of AFP-NHCC. These markers include PA [33], D-Dimer [33], C-reactive protein [34], platelet-lymphocyte ratio [34], lactate dehydrogenase [35] and GGT [35]. However, the predictive roles of FPR and GPR in AFP-NHCC remained unknown. Therefore, this study investigated FPR and GPR to assess whether these parameters related to the progression of AFP-NHCC and could have diagnostic value.

Research has proven that coagulation and nutritional status may affect the progression of HCC patients. Fibrinogen, as an important coagulation factor, synthesized by hepatocytes. Zhu et al. [36] demonstrated that the mRNA levels of fibrinogen were elevated both in cell lines and tissues, and increased plasma fibrinogen levels were associated with tumor thrombosis. On the other hand, gathering evidences have suggested that prealbumin is decreased and closely related with various malignancies, including liver cancer [37]. Therefore, the levels of FPR may be up-regulated in cancer patients. Indeed, preoperative FPR levels in the AFP-NHCC group were significantly higher than those in other groups, which concurred with previous reports. Sun et al. [20] reported that circulating FPR was significantly higher in patients harboring colorectal cancer than in benign and healthy subjects. Wang et al. [38] displayed that the levels of the prealbumin to fibrinogen ratio were reduced in severe acute pancreatitis and inversely proportional to the progression of acute pancreatitis. Another study by Zhang found that patients with low FPRs were observed to have a long survival, and the prognosis of stage III FPR-low gastric cancer patients undergoing chemotherapy was significantly superior to the patients without chemotherapy treatment [26]. FPR was also a prognostic marker in HCC, with a high FPR related to decreased survival and longer overall survival [27]. Moreover, several studies revealed that preoperative FPR was significantly correlated with clinical parameters in various solid tumors [20, 26]. Two scholars, $\mathrm{Hu}$ [39] and Zhang [27], found that FPR levels were higher in patients with large tumor sizes and advanced stages of HCC. The present study also demonstrated that FPR moderately increased with tumor size and BCLC stage of AFP-NHCC, demonstrating that this biological indicator may be related to the invasive phenotype of the disease.

As a crucial enzyme in glutathione metabolism, Gamma-glutamyl transferase (GGT) was continually 
elevated in metabolic-induced hepatic injury [40], was observed in this study. Salvatore et al. [41] demonstrated that the level of serum GGT elevated with the process of liver carcinogenesis and promoted tumor progression in an HCC animal model of male Wistar rats. Serum levels of GGT could also help with the selection of further treatment and clinical outcomes for patients with HCC [42]. Carr et al. [43] found that patients with significantly high GGT values were prone to poor overall survival in cases of low AFP HCC. Thrombocytopenia frequently occurs in chronic liver disease, mainly because of accelerated platelet destruction caused by hypersplenism and hepatocyte damage leading to a decrease in thrombopoietin [17]. These two opposite variables, GGT and PLT, were applied to GPR to further amplify and improve its predictive value. Park et al. [44] found that the relative risk of HCC development in the low-GPR group was lower than that of the high-GPR group. Compared to low-GPR patients, subjects with a higher GPR possessed a higher probability of cirrhosis, being a worse outcome [45]. The above studies indicate that GPR level increased with the severity of liver damage, which matches the findings of the present study as well. Our found that GPR levels were raised in the development of AFP-NHCC. In addition, regarding the relationship between GPR and clinical characteristics, it has been proven that patients with an elevated GPR had a higher probability of larger tumor size than individuals with a lower GPR [45]. Another study by $\mathrm{Hu}$ et al. [39] displayed that the levels of GPR were positively correlated with BCLC stages. Similar to previous studies, our results indicated that GPR levels increased with the progression of tumor size and cancer stage, including both the Edmondson-Steiner grade and BCLC stage.

The present study first assessed the preoperatory FPR and GPR levels to evaluate their diagnostic efficacy in the development of AFP-NHCC. Both FRP and GPR significantly increased with the progression of AFP-NHCC as well as tumor size and cancer stage. Moreover, high FPR and GPR levels were also independent poor outcome predictors in multivariate logistic regression analyses adjusted for potential predictors. These suggested that FPR and GPR might be important markers in the progression of patients with AFP-NHCC. FPR and GPR had good AUCs and sensitivity in identifying AFP-NHCC patients from controls. Many papers indicated that a series of biomarkers could help diagnose AFP-NHCC, showing the AUC of GP73 [46], AFP-L3 [46], and PIVKAII [47] used to distinguish AFP-NHCC from controls was $0.7811,0.6094$, and 0.856 , respectively, while the sensitivity was $66 \%, 50 \%$, and $76.3 \%$, respectively. These AUC values were lower than FPR and GPR, and the sensitivity of FPR was superior to those biomarkers for detecting AFP-NHCC. In addition, the combination of FPR and GPR had a larger AUC than FPR or GPR alone for discriminating AFP-NHCC patients and healthy controls. One retrospective study by Best and colleagues revealed that the detection rate of a combination of PIVKAII and AFP-L3 was only $68.4 \%$ for patients with AFPNHCC, which is lower than FPR and GPR combined. Zhang et al. [46] reported that the sensitivity achieved when using a combination of the assay results of AFP-L3 and GP73 were merely $40 \%$, which was not as sensitive as the combination with FPR and GPR. Compared to the $\mathrm{CHB}$ group, the AUC and sensitivity of PIVKAII in AFPNHCC patients was 0.73 and $51.02 \%$, respectively, which was slightly lower than our results $(0.745,67.22 \%)$. In addition, FPR and GPR were also moderate predictors for distinguishing AFP-NHCC patients from AFP-negative LC or AFP- negative $\mathrm{CH}$. Hence, the current results indicate that the combined use of FPR and GPR may improve the clinical diagnostic efficiency of differentiating AFPNHCC from other groups.

Several limitations should be concerned. First, we failed to distinguish viral physical status and viral load from the stratified analyses due to the relatively small sample size. Second, AFP-negative cases were limited and all came from the same hospital, which may create bias in evaluating the predictive value of these markers. Third, personal information, including dietary habits and family histories, was not obtained, which may influence the final results. Hence, future prospective studies require multiple centers, a larger scale, and more detailed information to validate these results.

\section{Conclusion}

The present study demonstrated that FPR and GPR were correlated with AFP-NHCC as well as tumor size and BCLC stage. The combination of FPR and GPR, as economic, simple, effective, and promising biomarkers, possessed a high diagnostic efficiency in the progression of patients with AFP-NHCC, especially in patients with early or small AFP-NHCC.

\section{Abbreviations \\ WBC: White blood cells; Hb: Hemoglobin; PLT: Platelets; TBIL: Total biliru- bin; ALT: Alanine aminotransferase; AST: Aspartate amino transferase; Fib: Fibrinogen; PA: Prealbumin; ALP: Alkaline phosphatase; GGT: Gamma-glutamyl transpeptidase; ROC: Receiver-operating characteristic curve; AUC: Area under curve; OR: Odd ratio; Cl: Confidence interval; FPR: Fibrinogen to prealbumin ratio; GPR: Gamma-glutamyl transpeptidase to platelet ratio; AFP: Alpha- fetoprotein; LC: Liver cirrhosis; CH: Chronic hepatitis; HCC: Hepatocellular carcinoma.}

Acknowledgements

We thank Scribendi.com for its linguistic assistance during the preparation of this manuscript. 


\section{Authors' contributions}

$\mathrm{LH}$ and ZN contributed equally to this work and should be considered as co-first authors. SL designed the study, LH performed the operation and wrote the manuscript, ZN performed the statistical analysis and revised the manuscript, LY and ZJ collected the data, SZ and XQ summarized the data. All authors read and approved the final manuscript.

\section{Funding}

This work was supported by the Self-financing Scientific Research Subject of Guangxi Health Department (Z20170315) and the Youth Science Foundation of The People's Hospital of Guangxi Zhuang Autonomous Region (QN2018-7).

\section{Availability of data and materials}

The datasets supporting the conclusions of this article is included within the article.

\section{Ethics approval and consent to participate}

The present study was approved by the Ethics Committee of the First Affiliated Hospital of Guangxi Medical University, and informed consent was obtained from all the participants.

\section{Consent for publication}

Not applicable.

\section{Competing interests}

The authors report no competing interests.

\section{Author details}

${ }^{1}$ Department of Clinical Laboratory, First Affiliated Hospital of Guangxi Medical University, Nanning 530021, Guangxi Zhuang Autonomous Region, China ${ }^{2}$ Department of Blood Transfusion, The People's Hospital of Guangxi Zhuang Autonomous Region, Nanning, Guangxi Zhuang Autonomous Region, China.

Received: 28 December 2019 Accepted: 29 February 2020

Published online: 12 March 2020

\section{References}

1. Bray F, Ferlay J, Soerjomataram I, Siegel RL, Torre LA, Jemal A. Global cancer statistics 2018: GLOBOCAN estimates of incidence and mortality worldwide for 36 cancers in 185 countries. CA Cancer J Clin. 2018;68(6):394-424

2. Tang A, Hallouch O, Chernyak V, Kamaya A, Sirlin CB. Epidemiology of hepatocellular carcinoma: target population for surveillance and diagnosis. Abdom Radiol. 2018:43(1):13-25.

3. Marrero JA, El-Serag HB. Alpha-fetoprotein should be included in the hepatocellular carcinoma surveillance guidelines of the American Association for the Study of Liver Diseases. Hepatology. 2011;53(3):1060-1 (author reply 1061-1062).

4. Taketa K. Alpha-fetoprotein: reevaluation in hepatology. Hepatology. 1990;12(6):1420-32

5. She $S$, Xiang $Y$, Yang $M$, Ding $X$, Liu $X, M a ~ L$, et al. C-reactive protein is a biomarker of AFP-negative HBV-related hepatocellular carcinoma. Int J Oncol. 2015:47(2):543-54.

6. Lo GH. Updated management of hepatocellular carcinoma. Hepatology. 2011;54(3):1113

7. Monfardini L, Orsi F, Caserta R, Sallemi C, Della Vigna P, Bonomo G, et al. Ultrasound and cone beam CT fusion for liver ablation: technical note. Int J Hyperth. 2018;35(1):500-4.

8. Tsuchiya N, Sawada Y, Endo I, Saito K, Uemura Y, Nakatsura T. Biomarkers for the early diagnosis of hepatocellular carcinoma. World J Gastroenterol. 2015;21(37):10573-83

9. Chen ZZ, Huang L, Wu YH, Zhai WJ, Zhu PP, Gao YF. LncSox4 promotes the self-renewal of liver tumour-initiating cells through Stat3-mediated Sox4 expression. Nat Commun. 2016;7:12598.

10. Dai M, Chen X, Liu X, Peng Z, Meng J, Dai S. Diagnostic value of the combination of golgi protein 73 and alpha-fetoprotein in hepatocellular carcinoma: a meta-analysis. PLoS ONE. 2015;10(10):e0140067.
11. Xu D, Su C, Sun L, Gao Y, Li Y. Performance of serum glypican 3 in diagnosis of hepatocellular carcinoma: a meta-analysis. Ann Hepatol. 2019:18(1):58-67.

12. Xing H, Zheng YJ, Han J, Zhang H, Li ZL, Lau WY, et al. Protein induced by vitamin $\mathrm{K}$ absence or antagonist-II versus alpha-fetoprotein in the diagnosis of hepatocellular carcinoma: a systematic review with metaanalysis. Hepatobiliary Pancreat Dis Int. 2018;17(6):487-95.

13. Lyra-Gonzalez I, Flores-Fong LE, Gonzalez-Garcia I, Medina-Preciado D, Armendariz-Borunda J. MicroRNAs dysregulation in hepatocellular carcinoma: insights in genomic medicine. World J Hepatol. 2015;7(11):1530-40.

14. Zheng RR, Huang M, Jin C, Wang HC, Yu JT, Zeng LC, et al. Cervical cancer systemic inflammation score: a novel predictor of prognosis. Oncotarget. 2016;7(12):15230-42.

15. Nagel T, Klaus F, Ibanez IG, Wege H, Lohse A, Meyer B. Fast and facile analysis of glycosylation and phosphorylation of fibrinogen from human plasma-correlation with liver cancer and liver cirrhosis. Anal Bioanal Chem. 2018:410(30):7965-77.

16. Shimura T, Shibata M, Kofunato Y, Okada R, Ishigame T, Kimura T, et al. Clinical significance of serum transthyretin level in patients with hepatocellular carcinoma. ANZ J Surg. 2018;88(12):1328-32.

17. Kurokawa T, Ohkohchi N. Platelets in liver disease, cancer and regeneration. World J Gastroenterol. 2017;23(18):3228-39.

18. Zhang JB, Chen Y, Zhang B, Xie X, Zhang L, Ge N, et al. Prognostic significance of serum gamma-glutamyl transferase in patients with intermediate hepatocellular carcinoma treated with transcatheter arterial chemoembolization. Eur J Gastroenterol Hepatol. 2011;23(9):787-93.

19. Liu DP, Lu W, Zhang ZQ, Wang YB, Ding RR, Zhou XL, et al. Comparative evaluation of GPR versus APRI and FIB-4 in predicting different levels of liver fibrosis of chronic hepatitis B. J Viral Hepatitis. 2018;25(5):581-9.

20. Sun F, Peng HX, Gao QF, Li SQ, Zhang J, Chen QG, et al. Preoperative circulating FPR and CCF score are promising biomarkers for predicting clinical outcome of stage II-III colorectal cancer patients. Cancer Manag Res. 2018;10:2151-61.

21. Sahni A, Francis CW. Vascular endothelial growth factor binds to fibrinogen and fibrin and stimulates endothelial cell proliferation. Blood. 2000;96(12):3772-8

22. Simpson-Haidaris PJ, Rybarczyk B. Tumors and fibrinogen. The role of fibrinogen as an extracellular matrix protein. Ann NY Acad Sci. 2001:936:406-25.

23. Zhang $X$, Long $Q$. Elevated serum plasma fibrinogen is associated with advanced tumor stage and poor survival in hepatocellular carcinoma patients. Medicine. 2017;96(17):e6694.

24. Douville P, Talbot J, Lapointe R, Belanger L. Potential usefulness of serum prealbumin in total parenteral nutrition. Clin Chem. 1982;28(7):1706-7.

25. Yang F, Wei L, Huo X, Ding Y, Zhou X, Liu D. Effects of early postoperative enteral nutrition versus usual care on serum albumin, prealbumin, transferrin, time to first flatus and postoperative hospital stay for patients with colorectal cancer: a systematic review and meta-analysis. Contemp Nurse. 2018;54(6):561-77.

26. Zhang J, Li SQ, Liao ZH, Jiang YH, Chen QG, Huang B, et al. Prognostic value of a novel FPR biomarker in patients with surgical stage II and III gastric cancer. Oncotarget. 2017;8(43):75195-205.

27. Zhang L, Chen QG, Li SQ, Zhang J, Min QH, Gao QF, et al. Preoperative fibrinogen to prealbumin ratio as a novel predictor for clinical outcome of hepatocellular carcinoma. Future Oncol. 2019;15(1):13-22.

28. Lemoine M, Shimakawa Y, Nayagam S, Khalil M, Suso P, Lloyd J, et al. The gamma-glutamyl transpeptidase to platelet ratio (GPR) predicts significant liver fibrosis and cirrhosis in patients with chronic HBV infection in West Africa. Gut. 2016:65(8):1369-76.

29. Wang J, Yan $X$, Yang $Y$, Chang $H$, Jia B, Zhao XA, et al. A novel predictive model using routinely clinical parameters to predict liver fibrosis in patients with chronic hepatitis B. Oncotarget. 2017;8(35):59257-67.

30. Ke MY, Zhang M, Su Q, Wei S, Zhang J, Wang Y, et al. Gamma-glutamyl transpeptidase to platelet ratio predicts short-term outcomes in hepatocellular carcinoma patients undergoing minor liver resection. J Surg Res. 2018:231:403-10.

31. Song $P$, Tang $Q$, Feng $X$, Tang W. Biomarkers: evaluation of clinical utility in surveillance and early diagnosis for hepatocellular carcinoma. Scand J Clin Lab Invest Suppl. 2016:245:S70-6. 
32. Song P, Tobe RG, Inagaki Y, Kokudo N, Hasegawa K, Sugawara Y, et al. The management of hepatocellular carcinoma around the world: a comparison of guidelines from 2001 to 2011. Liver Int. 2012;32(7):1053-63.

33. Jing W, Peng R, Zhu M, Lv S, Jiang S, Ma J, et al. Differential expression and diagnostic significance of pre-albumin, fibrinogen combined with DDimer in AFP-negative hepatocellular carcinoma. Pathol Oncol Res. 2019. https://doi.org/10.1007/s12253-019-00752-8.

34. Suner A, Carr BI, Akkiz H, Karakulah G, Uskudar O, Yalcin K, et al. C-reactive protein and platelet-lymphocyte ratio as potential tumor markers in lowalpha-fetoprotein hepatocellular carcinoma. Oncology. 2019;96(1):25-32.

35. Wang X, Mao M, He Z, Zhang L, Li H, Lin J, et al. Development and validation of a prognostic nomogram in AFP-negative hepatocellular carcinoma. Int J Biol Scil. 2019;15(1):221-8.

36. Zhu WL, Fan BL, Liu DL, Zhu WX. Abnormal expression of fibrinogen gamma (FGG) and plasma level of fibrinogen in patients with hepatocellular carcinoma. Anticancer Res. 2009;29(7):2531-4.

37. Qiao W, Leng F, Liu T, Wang X, Wang Y, Chen D, et al. Prognostic value of prealbumin in liver cancer: a systematic review and meta-analysis. Nutr Cancer. 2019. https://doi.org/10.1080/01635581.2019.1661501.

38. Yue W, Liu Y, Ding W, Jiang W, Huang J, Zhang J, et al. The predictive value of the prealbumin-to-fibrinogen ratio in patients with acute pancreatitis. Int J Clin Pract. 2015;69(10):1121-8.

39. Hu Z, Chen H, Chen S, Huang Z, Qin S, Zhong J, et al. The value of neutrophil to lymphocyte ratio and gamma-glutamyl transpeptidase to platelet ratio in patients with hepatocellular carcinoma. Medicine. 2019;98(9):e14749.

40. Petta S, Macaluso FS, Barcellona MR, Camma C, Cabibi D, Di Marco $V$, et al. Serum gamma-glutamyl transferase levels, insulin resistance and liver fibrosis in patients with chronic liver diseases. PLOS ONE. 2012;7(12):e51165.
41. Moreira AJ, Rodrigues GR, Bona S, Fratta LX, Weber GR, Picada JN, et al. Ductular reaction, cytokeratin 7 positivity, and gamma-glutamyl transferase in multistage hepatocarcinogenesis in rats. Protoplasma. 2017;254(2):911-20.

42. Wang Z, Song P, Xia J, Inagaki Y, Tang W, Kokudo N. Can gamma-glutamyl transferase levels contribute to a better prognosis for patients with hepatocellular carcinoma? Drug Discov Ther. 2014;8(3):134-8.

43. Carr BI, Guerra V, Giannini EG, Farinati F, Ciccarese F, Rapaccini GL, et al. Low alpha-fetoprotein HCC and the role of GGTP. Int J Biol Mark. 2014;29(4):e395-402

44. Park YE, Kim BK, Park JY, Kim DY, Ahn SH, Han KH, et al. Gamma-glutamyl transpeptidase-to-platelet ratio is an independent predictor of hepatitis $B$ virus-related liver cancer. J Gastroenterol Hepatol. 2017;32(6):1221-9.

45. Pang Q, Bi JB, Wang ZX, Xu XS, Qu K, Miao RC, et al. Simple models based on gamma-glutamyl transpeptidase and platelets for predicting survival in hepatitis B-associated hepatocellular carcinoma. OncoTargets Therapy. 2016;9:2099-109.

46. Zhang Z, Zhang Y, Wang Y, Xu L, Xu W. Alpha-fetoprotein-L3 and Golgi protein 73 may serve as candidate biomarkers for diagnosing alphafetoprotein-negative hepatocellular carcinoma. OncoTargets Therapy. 2016;9:123-9.

47. Ji J, Wang H, Li Y, Zheng L, Yin Y, Zou Z, et al. Diagnostic evaluation of Desgamma-carboxy prothrombin versus alpha-fetoprotein for hepatitis $B$ virus-related hepatocellular carcinoma in China: a large-scale, multicentre study. PLoS ONE. 2016;11(4):e0153227.

\section{Publisher's Note}

Springer Nature remains neutral with regard to jurisdictional claims in published maps and institutional affiliations.
Ready to submit your research? Choose BMC and benefit from:

- fast, convenient online submission

- thorough peer review by experienced researchers in your field

- rapid publication on acceptance

- support for research data, including large and complex data types

- gold Open Access which fosters wider collaboration and increased citations

- maximum visibility for your research: over $100 \mathrm{M}$ website views per year

At BMC, research is always in progress.

Learn more biomedcentral.com/submissions 